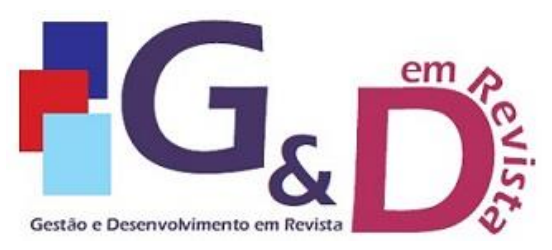

Gestão e Desenvolvimento em Revista V. 6, N. 1, jan-jun/2020, p. 60-76. ISSN online: $2446-8738$

Artigo recebido em: $11 / 05 / 2020$

Artigo aprovado em: 28/06/2020

\title{
TÁTICAS VINCULANTES: UM ESTUDO BIBLIOMÉTRICO E ANALÍTICO SOBRE PESQUISAS FUTURAS
}

\author{
Vanessa Teresinha Rigotti \\ Graduação em Administração - Comércio Exterior. Mestrado em Administração. Atualmente é \\ professora na Universidade de Caxias do Sul (UCS). \\ E-mail: vtrigott@ucs.br
}

Daniel Hank Miri

Graduação e Mestrado em Administração. Atualmente é professora na Universidade de Caxias do Sul (UCS).E-mail: dhmiri@ucs.br

Juliana Matte

Graduacão e Mestrado em Administração. Doutoranda em Administração pela Universidade de Caxias do Sul (UCS).

E-mail: ju.cxs1@gmail.com

\section{Resumo}

Quando as empresas buscam em suas estratégias maneiras de implementar ações que gerem os fatores de transformação comercial, elas estão se utilizando de táticas vinculantes que podem ser três: Financeira, Social e Estrutural. As táticas de vínculo ou ligação foram abordadas inicialmente pela psicologia e algumas décadas depois associadas ao marketing para avaliar os aspectos psicológicos, emocionais, econômicos ou físicos e estruturais intrínsecos a um relacionamento. Dessa forma, as táticas vinculantes são associadas como responsáveis para obtenção de satisfação e retenção de clientes. Nesse sentido, o objetivo deste artigo foi analisar o conceito de táticas vinculantes, identificando campos e gaps de pesquisa a serem explorados. Uma análise bibliométrica foi realizada em cinco bases de dados de abrangência internacional com a análise qualitativa dos artigos científico relevantes. Os resultados encontrados indicaram que os estudos pesquisados comprovam que as táticas vinculantes financeiras, sociais e estruturais impactam positivamente na confiança, no compromisso, na qualidade de relacionamento, no custo de troca, no valor percebido, na intenção e comportamento do consumidor, na satisfação do cliente, que associados geram a retenção do cliente.

Palavras-chave: Táticas Vinculantes. Relacionamento com os Clientes. Bibliometria.

\section{Abstract}

When companies seek in their strategies ways to implement actions that generate the factors of commercial transformation, they are using binding tactics that can be three: Financial, Social and Structural. Bonding tactics were first approached by psychology and a few decades later associated with marketing to assess the psychological, emotional, economic or physical and structural aspects intrinsic to a relationship. In this way, binding tactics are associated as responsible for obtaining customer satisfaction and retention. In this sense, the objective of this article was to analyze the concept of binding tactics, identifying fields and research gaps to be explored. A bibliometric analysis was carried out in five databases of international scope with a qualitative analysis of the relevant scientific articles. The results found indicated that the researched studies prove that the financial, social and structural binding tactics positively impact confidence, commitment, quality of relationship, cost of exchange, perceived value, consumer intention and behavior, customer satisfaction which associates generate customer retention.

Keywords: Bonding Tactics. Relationship with Customer. Bibliometric. 


\section{INTRODUÇÃO}

O marketing de relacionamento surgiu por meio de Leonardo Berry (1983), cujo autor extraiu do marketing de serviços esse conceito como o processo de "atração, manutenção e aumento do relacionamento com clientes".

Através do marketing de relacionamento ocorreram mudanças de paradigma dos conceitos de marketing desenvolvidos desde os anos 1960, porque propunha uma nova filosofia empresarial na qual é tão importante fazer negócios quanto gerenciá-los organizacionalmente. Embora recente, 0 gerenciamento de relacionamento com os clientes se tornou uma prática fundamental para as organizações que querem conhecer melhor os seus clientes para melhor atender e assim retê-los (BERRY, 1983).

A alta competitividade do mercado fez com que as empresas iniciassem verdadeiras batalhas para conquistar seus consumidores, e com o passar dos anos conquistar novos consumidores se tornou igualmente importante a mantê-los, porque conforme as pesquisas de Vavra (1994), a retenção de clientes é a chave para o sucesso no mercado do novo milênio.

Para Edward e Sahadev (2011), o alto custo de aquisição de novos clientes comparado com um custo menor em reter os clientes já existentes faz com que as empresas se esforcem cada vez mais em aumentar sua rentabilidade através da retenção de clientes. A retenção de clientes, conforme Lopes et al. (2012), pode ocorrer por diversos fatores: financeiros, sociais e estruturais, que são variáveis que interferem na lealdade do cliente, na confiança, no comprometimento e influenciam diretamente a duração do relacionamento.

Os fatores acima citados são considerados vínculos que a empresa tem para com o cliente criando um valor de uso para o relacionamento. Quando a empresa cria vínculos com seus clientes por meio de cláusulas contratuais que geram altos custos na troca de fornecedor, descontos expressivos, taxas de juros mais baixas, programas de benefícios e fidelidade, está utilizando a tática vinculante financeira (LIANG; WANG, 2007b).

Mimouni-Chaabane e Volle (2010), ao estudarem benefícios simbólicos, notaram que alguns clientes sentem a necessidade de fazer parte de um grupo seleto e exclusivo, em que possam desfrutar de ofertas exclusivas, diferenciação e aprovação social. Nesses casos em que os custos de troca são barreiras emocionais e laços sociais têm-se a tática vinculante social. Se apresentam, segundo Rodriguez e Wilson (2002), como táticas vinculantes estruturais quando investimentos funcionais, estratégicos e até mesmo econômicos geram benefícios explícitos tão grandes, que se tornam barreiras para mudar de fornecedor.

Muitas empresas lançam mão de estratégias para criar a retenção de seus clientes, que é quando a intenção desses é continuar comprando produtos ou serviços a ponto de ocorrer muitas vezes a fidelização e lealdade. Essas estratégias podem ser descritas como táticas que criam vínculos, entre empresas e clientes, de natureza econômica, emocional ou estrutural.

O objetivo deste estudo foi analisar o conceito de táticas vinculantes, identificando campos e gaps de pesquisa a serem explorados. Para tal, procedeu-se uma bibliometria, analisando os artigos publicados na base Scopus, Sage, Emerald, Science Direct e Web of Science. A partir dos resultados encontrados, foi elaborada uma caracterização dos estudos quanto a sua origem, periódico de publicação e método desenvolvido. Após essa análise inicial, procedeu-se a análise das temáticas 
desenvolvidas nos estudos e a discussão dos resultados encontrados, finalizando com as conclusões.

\section{REFERENCIAL TEÓRICO}

As táticas vinculantes se originam dos estudos dos psicólogos McCall e Turner, em 1970, que caracterizam o vínculo de relacionamento "como anexos de relações psicológicas, emocionais, econômicas ou físicas em um relacionamento que são fomentados por associação e interação e servem para unir as partes sob relações relacionais" (YUFANG; XIAOBING, 2012, p. 95).

Wilson e Venkatapparao (1986) discutiram o vínculo nas relações na empresa, entre comprador e vendedor, conceituando relacionamentos de longo prazo. Os autores descreveram que um intenso relacionamento comercial levaria sete anos para desfazer completamente os laços existentes entre eles, pois esses laços exigem que ambos façam adequações entre eles em produtos, instalações físicas e recursos humanos (ARANTOLA, 2002).

Han (1991) identificou dois tipos de ligação táticas: ligação social, cuja empresa cria laços com os clientes a partir das relações sociais com os funcionários e vínculo estrutural, cujo laço se dá em função de fatores financeiros, estratégicos ou organizacionais. No mesmo ano, Berry e Parasuraman (1991) também pesquisaram sobre as táticas vinculantes, acrescentando a percepção financeira, além da social e da estrutural. O vínculo financeiro é a tática em que as empresas incentivam os clientes a consumir mais e manter relacionamentos de longo prazo por preço ou estímulos financeiros, a fim de aumentar a frequência de compra do cliente ou alcançar sua retenção (BERRY, 1995).

A partir desse estudo, as pesquisas seguintes continuaram mencionando essas mesmas três práticas, como Peltier e Westfall (2000), Lin, Weng e Hsieh (2003), Chiu et al. (2005), Hsieh, Chiu e Chiang (2005) e Wang, Liang e Wu (2006). Conforme a Figura 1, que se baseia na conceituação dos autores Liang e Wang (2008), os clientes podem ser retidos por meio da criação desses três vínculos, que geram confiança, que por sua vez gera o comprometimento e que pode se tornar lealdade.

Figura 1 - Modelo Conceitual de Relacionamento

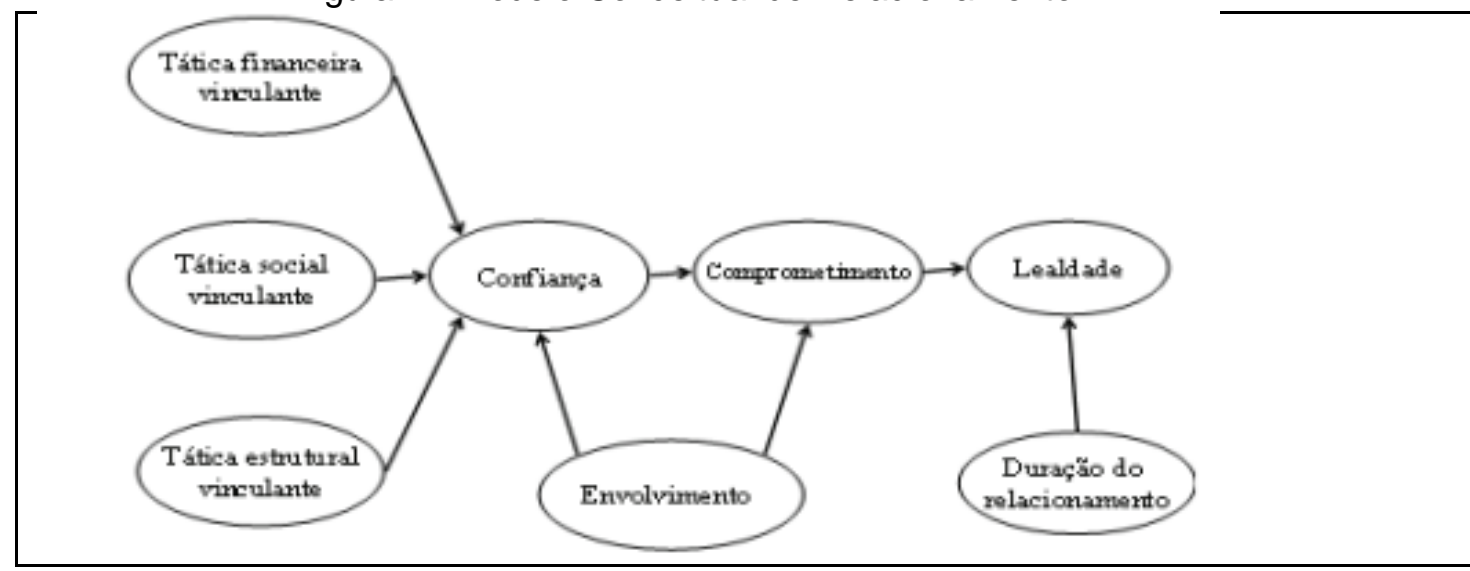

Fonte: Liang e Wang (2008).

Após o modelo conceitual de relacionamento seguem as três táticas vinculantes. 


\subsection{TÁTICA FINANCEIRA}

A tática vinculante financeira consiste em fornecer preço ou estímulo financeiro para incentivar um cliente a consumir mais produtos ou serviços para que a empresa possa estabelecer um relacionamento de longo prazo (BERRY, 1995). Dessa forma, a empresa pode ter consequências positivas tanto para os vendedores (que aumentam suas vendas), quanto para os compradores (percebendo que a compra possui uma utilidade de aquisição melhorada) através de benefícios mútuos (BURNHAM; FRELS; MAHAJAN, 2003; KOTLER; KELLER, 2012).

Para Verhoef (2003), tanto a retenção quanto o desenvolvimento dos clientes são afetados positivamente pelos incentivos econômicos. Esses incentivos podem ser programas de frequência de compras, os quais são conhecidos como programas de fidelidade, que acumulam pontos que são trocados por mercadorias, benefícios adicionais e serviços (LEENHEER; BIJMOLT, 2008).

Benefícios financeiros advindos dos chamados programas de fidelidade, segundo Mimouni-Chaabane e Volle (2010), proporcionam ao cliente menor gasto, essa redução dos gastos advém de serviços agregados e conveniência comercial pela economia de tempo e esforço. Benefícios financeiros oriundos de programas de fidelização, para Verhoef (2003), impactam positivamente na duração do relacionamento com a empresa e aumenta a quota de clientes.

Quanto maior forem os custos de mudança maior serão os índices de retenção dos clientes (EDWARD; SAHADEV, 2011). Dissolução de parcerias ou quebras de contrato podem ter um custo elevado fazendo com que o cliente continue atrelado ao fornecedor mesmo estando insatisfeito. Desta forma, os benefícios e os ganhos mútuos advindos da relação entre as partes envolvidas devem ser claramente maiores que os da atuação isolada, servindo como elemento de incentivo à manutenção do relacionamento (WANG; LIANG; WU, 2006).

Uma espécie de vínculo com o cliente pode ser a motivação de consumo ou utilização por meio de decisão de preços, envolvendo descontos de preço, melhores taxas de juros, entre outros (LIANG; WANG, 2007b). Dessa forma, as táticas vinculantes financeiras podem ser classificadas em dois subgrupos: um ligado à economia e outro aos benefícios da compra (DONG; ZHANG; YANG, 2009). A primeira faz ofertas de preços especiais, descontos, cupons e preços mais baixos, enfatizando a manutenção do cliente a partir da redução de custos e a segunda por sua vez oferece presentes aos clientes, pontos cumulativos em sistemas de fidelidade, benefícios por compras em maior quantidade de uma só vez, além de brindes diferentes por tipos diferentes aquisições, enfatizando a manutenção do cliente a partir de concessões "especiais" (DONG; ZHANG; YANG, 2009).

As táticas vinculantes financeiras, para Liang e Wang (2007b), são uma espécie de estímulo ou de motivação para o consumo por meio da decisão por preços, tais como descontos, taxa de juros, benefícios quantificáveis.

\subsection{TÁTICA SOCIAL}

As relações interpessoais referem-se aos vínculos pessoais que são desenvolvidos entre os clientes e os fornecedores dos serviços, por meio de seus empregados, individualizando e personalizando 0 tratamento de clientes 
preferenciais (KOTLER; KELLER, 2012). Para Narteh et al. (2013), os vínculos sociais exercem influência significativa na fidelidade do cliente. Em seu estudo, Mimouni-Chaabane e Volle (2010), tratam os benefícios simbólicos como uma necessidade de o cliente sentir-se especial e fazer parte de um grupo exclusivo. $O$ vínculo social pode ser obtido com práticas de marketing de relacionamento adicionais como convites para o cliente participar de eventos especiais (PAULSSEN; ROULET; WILKE, 2014). As táticas vinculantes sociais são tratadas, por Turner (1970), como ligações ou vínculos pessoais construídos durante a interação entre as partes.

Sob a ótica das empresas, as táticas vinculantes Sociais enfatizam principalmente a manutenção de relações estreitas com clientes, entendendo suas necessidades, seus desejos, suas demandas e suas expectativas, de modo que se desenvolvam relações interpessoais ou sociais, emergindo amizade e até mesmo afeto (YUFANG; XIAOBING, 2012). Ou seja, as táticas vinculantes sociais dizem respeito às atividades que desenvolvem e mantêm relacionamentos de longo prazo com os clientes existentes por meio de foco no cliente e da criação de uma proximidade (ZHANG, 2005; DONG; ZHANG; YANG, 2009).

As táticas vinculantes sociais enfatizam, principalmente, a manutenção de relações estreitas com seus clientes. Tais táticas podem, ainda, aumentar a tolerância do cliente a eventuais erros ou falhas, dando à empresa a oportunidade de resolver o problema antes que a substitua por um concorrente (YUFANG; XIAOBING, 2012).

A construção de laços sociais é uma estratégia recomendada pelo marketing de relacionamento que objetiva gerar relações fortes (PALMATIER et al., 2006). No entanto, para os funcionários, a orientação para construir relações sociais com os clientes é considerado um pedido exigente (HENNIG-THURAU; GWINNER; GREMLER, 2002). Dessa forma, saber quando incentivar o desenvolvimento de laços sociais entre a empresa e os seus clientes é uma estratégia eficaz e altamente relevante.

Existem diversos custos de mudança relacionais, que são associados a barreiras psicológicas e emocionais, como laços sociais e pessoais. Esses vínculos impedem a mudança por comprometimento relacional $e$ afetivo, além de proporcionar atendimento personalizado, fazendo com que os consumidores fiquem satisfeitos e confiem na empresa, ajudando a criar elos, lealdade e comprometimento do cliente (NARTEH et al., 2013).

Assim, é possível sugerir que o relacionamento interpessoal constitui uma forma de barrar a saída dos clientes (JONES; MOTHERSBAUGH; BEATTY, 2000). Relacionamentos positivos, conforme Bernardon e Lopes (2018), podem ser considerados diferenciais para muitas empresas, porque resultando na satisfação dos consumidores, pode gerar fidelidade, e também, a indicação do produto ou serviço para um amigo ou colega.

\subsection{TÁTICA ESTRUTURAL}

As táticas vinculantes estruturais, conforme Liang e Wang (2008), são relacionados à estrutura, administração e institucionalização de normas em um relacionamento. Podem ser entendidos como as regras, as políticas, os procedimentos, a infraestrutura ou os acordos que fornecem estrutura formal para um relacionamento. 
As normas ou rotinas que informalmente governam a interação e os sistemas e tecnologias organizacionais que permitem ou facilitam a interação podem fornecer laços psicológicos, legais e físicos que vinculam as partes a um relacionamento e dificultam a consideração de outros parceiros de negócio. Esse tipo de tática de ligação significa fornecer um programa de solução estrutural para os consumidores. Para Kotler e Keller (2012), essa tática objetiva proporcionar facilidade, conveniência e praticidade ao cliente.

Ao oferecer este tipo de vínculo as empresas podem consolidar a sua relação com os clientes e diferenciar-se fortemente dos concorrentes (LIANG; WANG, 2008). A tática estrutural, para Zhang, Dong e Zhang (2010), serve como uma espécie de motivação para o cliente permanecer na relação. Assim como serviços logísticos, que para Parasuraman e Zeithaml (2005), são processos de entrega de produtos que podem oferecer ao cliente maior conveniência e rapidez colocando a empresa muito à frente de seus concorrentes.

Para Oliver (2010), são altos os custos de troca de fornecedor para clientes acostumados a terem a sua disposição uma gama estrutural de atendimento. Embora os vínculos estruturais possam ser insuficientes para a continuidade do relacionamento em razão da presença de elos fracos de vínculo social e dar margem à incidência de comportamentos oportunistas (MADHOK; 1995; WULF; ODEKERKENSCHRÖDER; IACOBUCCI, 2001), o estudo de Wang, Liang e Wu (2006) apresenta as táticas vinculantes estruturais com maior poder de explicação sobre a qualidade do relacionamento, seguidas das táticas vinculantes sociais.

\section{MÉTODO DE PESQUISA}

O estudo possui abordagem qualitativa, realizada por meio da análise e interpretação dos artigos científicos de maior relevância (LAKATOS; MARCONI, 2011). Contudo, para a identificação dos artigos a serem investigados, realizou-se uma análise bibliométrica, que é um método flexível para avaliar a tipologia, a quantidade e a qualidade das fontes de informação citadas em pesquisas. $O$ produto da análise bibliométrica são os indicadores científicos dessa produção. Conforme Figueiredo (1977), a bibliometria possui uma dupla preocupação: a análise da produção científica e a busca de benefícios práticos imediatos para bibliotecas.

A Lei de Lotka, formulada em 1926, foi construída a partir de um estudo sobre a produtividade de cientistas em que foi realizada a contagem de autores presentes e descobriu-se que uma larga proporção da literatura científica é produzida por um pequeno número de autores, e que unindo toda a produção de vários pequenos autores havia uma igualdade de produção.

A segunda lei bibliométrica, de Bradford, incide sobre conjuntos de periódicos. Em 1934, com o objetivo de descobrir a extensão na qual artigos de um assunto científico específico apareciam em periódicos destinados a outros assuntos, estudando a distribuição dos artigos em termos de variáveis de proximidade ou de afastamento e formula a Lei da Dispersão. Bradford viu que era por essa razão que os índices tinham dificuldade para atingir a cobertura completa de assuntos. Ele constatou que mais da metade do total de artigos úteis não estavam sendo cobertos pelos serviços de indexação e resumos.

$\mathrm{Na}$ sequência Zipf formulou a terceira lei da bibliometria: Lei do Princípio do Menor Esforço, cujo conteúdo fala da existência da economia do uso de palavras. A tendência de usar o mínimo de palavras faz com que elas não se dispersem, pelo 
contrário, uma mesma palavra vai ser usada muitas vezes e as palavras mais usadas indicam o assunto do documento. Para isso, foi realizada uma busca nas bases de dados Scopus, Emerald, Sage, Science Direct e Web of Science, individualmente. O termo procurado foi "bonding tactics" entre aspas, sem um período de tempo específico e sem outras limitações.

Para a análise e interpretação dos artigos científicos de maior relevância foi utilizada a abordagem qualitativa exploratória. Realizou-se a revisão e descrição dos artigos (HENNINK et al., 2011) que relatam o conceito de bonding tactics.

\section{ANÁLISE E DISCUSSÃO DOS RESULTADOS}

Os resultados da pesquisa realizada são apresentados em duas etapas: a primeira etapa se constitui da apresentação da bibliometria e a segunda, são apresentadas as análises de conteúdo dos artigos.

\subsection{BIBLIOMETRIA}

A partir das consultas realizadas, em 06 de junho de 2019, foram localizados 1 registro na base Sage, 5 registros da base Emerald, 3 registros na base Science Direct, 12 registros na base Scopus e 7 registros na base Web Science, totalizando 23 registros referentes a esta temática, desconsiderando, para esse estudo, os artigos científicos da área da saúde. O Quadro 1, apresenta os artigos encontrados na pesquisa.

Quadro 1 - Artigos encontrados nas bases sobre "bonding tactics"

\begin{tabular}{|c|c|c|c|c|c|}
\hline $\mathrm{N}^{\circ}$ & Autores & Ano & Journal & Base & $\begin{array}{l}\text { Cita- } \\
\text { ções }\end{array}$ \\
\hline 1 & MILAN et al. & 2018 & Benchmarking & $\begin{array}{l}\text { Scopus; Web of } \\
\text { Science }\end{array}$ & 1 \\
\hline 2 & WANG. & 2018 & $\begin{array}{l}\text { Journal of } \\
\text { Marketing }\end{array}$ & $\begin{array}{l}\text { Scopus; Web of } \\
\text { Science }\end{array}$ & 1 \\
\hline 3 & HALIM. & 2018 & European Research Studies Journal & Scopus & 0 \\
\hline 4 & WANG et al. & 2016 & Journal of Business Research & \begin{tabular}{lrr} 
Scopus; & \multicolumn{2}{c}{ Science } \\
Direct; & Web of \\
Science & &
\end{tabular} & 9 \\
\hline 5 & $\begin{array}{l}\text { PAULSSEN; } \\
\text { ROULET; WILKE }\end{array}$ & 2014 & European Journal of Marketing & $\begin{array}{l}\text { Scopus; Web of } \\
\text { Science }\end{array}$ & 25 \\
\hline 6 & $\begin{array}{l}\text { YUFANG; } \\
\text { XIAOBING }\end{array}$ & 2012 & $\begin{array}{lll}\text { Service Sciences } & \text { (JCSS), } \\
\text { International Joint Conference on } & \end{array}$ & Scopus & 0 \\
\hline 7 & ZHANG & 2011 & $\begin{array}{l}\text { E-Business and E-Government (ICEE), } \\
2011 \text { International Conference on }\end{array}$ & Scopus & 0 \\
\hline 8 & $\begin{array}{l}\text { DONG; } \quad \text { ZHANG; } \\
\text { YANG }\end{array}$ & 2009 & $\begin{array}{l}\text { Management and Service Science, } \\
2009 . \quad \text { MASS'09. International } \\
\text { Conference on }\end{array}$ & Scopus & 0 \\
\hline 9 & LIANG; WANG & 2008 & Service Industries Journal & $\begin{array}{l}\text { Scopus; Web of } \\
\text { Science }\end{array}$ & 119 \\
\hline 10 & $\begin{array}{l}\text { LIANG; } \quad \text { CHEN; } \\
\text { WANG }\end{array}$ & 2008 & Service Industries Journal & $\begin{array}{l}\text { Scopus; Web of } \\
\text { Science }\end{array}$ & 14 \\
\hline 11 & LIANG; WANG & 2007 & Measuring Business Excellence & Scopus & 36 \\
\hline 12 & LIANG; WANG & 2006 & Service Industries Journal & $\begin{array}{l}\text { Scopus; Web of } \\
\text { Science }\end{array}$ & 127 \\
\hline 13 & LIANG; WANG & 2007 & International Journal of Bank Marketing & Emerald & 36 \\
\hline 14 & WANG & 2008 & Measuring Business Excellence & Emerald & 14 \\
\hline
\end{tabular}




\begin{tabular}{|c|l|l|l|l|c|}
\hline 15 & WANG; LIANG & 2010 & Measuring Business Excellence & Emerald & 4 \\
\hline 16 & $\begin{array}{l}\text { LIANG; WANG; } \\
\text { FARQUHAR }\end{array}$ & 2009 & International Journal of Bank Marketing & Emerald & 87 \\
\hline 17 & LAKSAMANA et al. & 2013 & Marketing Intelligence \& Planning & Emerald & 14 \\
\hline 18 & PARAHOO & 2012 & International Journal of Bank Marketing & Emerald & 16 \\
\hline 19 & SHI et al. & 2016 & Journal of Services Marketing & Emerald & 05 \\
\hline 20 & LEE & 2018 & $\begin{array}{l}\text { Asia Pacific Journal of Marketing and } \\
\text { Logistics }\end{array}$ & Emerald & 0 \\
\hline 21 & $\begin{array}{l}\text { KOUL; SINHA; } \\
\text { MISHRA }\end{array}$ & 2016 & Global Business Review & Sage & 02 \\
\hline 22 & DAVCIK; SHARMA & 2016 & Journal of Business Research & Science Direct & 21 \\
\hline 23 & WEI et al. & 2014 & Information \& Management & Science Direct & 13 \\
\hline
\end{tabular}

Fonte: Elaborado pelos autores (2020).

Ao analisar os artigos publicados e os anos de publicação é possível perceber na análise temporal a oscilação de interesse pelo assunto, sendo que em 2015 e 2017 nenhum artigo científico foi publicado, como pode ser visto no Gráfico 1 apresentado a seguir.

Figura 1 - Linha do tempo das publicações

\section{Artigos Publicador X Ano}

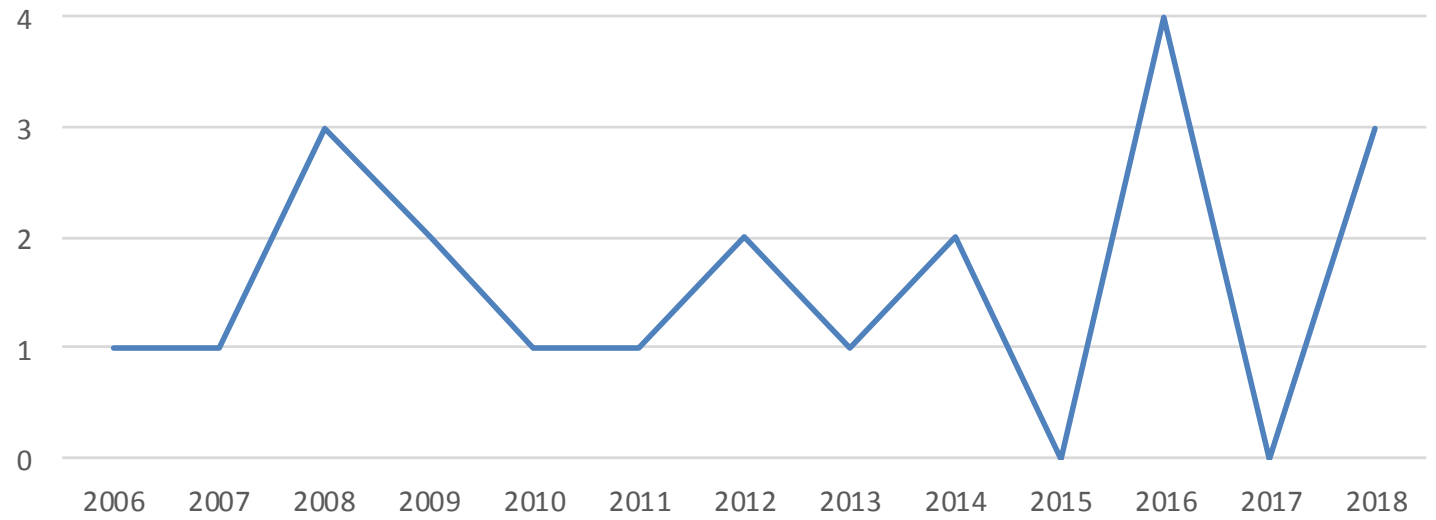

Fonte: Elaborado pelos autores (2020).

Analisando os artigos da presente pesquisa, em relação à Lei de Lotka, é possível constatar que, dos 23 artigos analisados, 8 artigos são de autoria de WenHung Wang, 6 artigos de Chiung-Ju Liang, 2 artigos de Rui-Xue Zhang e 3 artigos de Yonggui Wang. Logo, apenas 4 autores representam a produção científica de 12 artigos, sendo que os outros 30 autores são responsáveis apenas pela produção de 11 artigos. Mesmo que a produção seja quase equivalente, no volume total 12 para 11, a discrepância no volume de produção é enorme 4 autores produzem mais do que 30 autores somados.

Quanto a Lei de Bradford foi possível constatar que as revistas que mais publicaram sobre o Tema pesquisado foram Service Industries Journal, Measuring Business Excellence e International Journal of Bank Marketing, ambas com três artigos cada. A revista Journal of Business Research possui dois artigos publicados e as outras doze revistas apenas um artigo cada. 
Em relação a Lei de Zipf, também conhecida como Lei do Menor Esforço, dos 23 artigos estudados, 15 artigos apresentam ou no título, ou no resumo ou nas palavras-chave o termo "bonding Tactics" ou "bonding".

No presente estudo o que ficou bastante evidente foi o "Efeito Mateus", que segundo Merton (1968), aos que têm muito mais lhes será dado, e aos que têm pouco até isso lhes será tirado. Os pesquisadores que produzem muito e já têm muitas publicações conseguem publicar e até mesmo receber apoio e financiamento para realizar mais e os pesquisadores iniciantes, que por vezes tentam publicar e precisam de fomento para suas pesquisas não conseguem espaço e incentivo.

A partir do "Efeito Mateus" sobre a ciência, das três Leis da Bibliometria: Lotka, Bradford e Zipf e da triangulação entre a pesquisa quantitativa (bibliometria) e a qualitativa (análise minuciosa do conteúdo) foi criado o Quadro 2, que corresponde às principais obras e mais citadas sobre o tema proposto.

Quadro 2 - Artigos de maior relevância

\begin{tabular}{|l|l|l|l|c|}
\hline Autores & Ano & Título & Journal & Citações \\
\hline $\begin{array}{l}\text { LIANG; } \\
\text { WANG }\end{array}$ & 2006 & $\begin{array}{l}\text { The behavioural sequence of the } \\
\text { financial services industry in Taiwan: } \\
\text { Service quality, relationship quality and } \\
\text { behavioural loyalty }\end{array}$ & $\begin{array}{l}\text { Service } \\
\text { Industries } \\
\text { Journal }\end{array}$ & 133 \\
\hline $\begin{array}{l}\text { LIANG; } \\
\text { WANG }\end{array}$ & 2008 & $\begin{array}{l}\text { Does online relationship marketing } \\
\text { enhance customer retention and cross- } \\
\text { buying? }\end{array}$ & $\begin{array}{l}\text { Service } \\
\text { Industries } \\
\text { Journal }\end{array}$ & 119 \\
\hline $\begin{array}{l}\text { LIANG; } \\
\text { WANG; } \\
\text { FARQUHAR }\end{array}$ & 2009 & $\begin{array}{l}\text { The influence of customer perceptions } \\
\text { on financial performance in financial } \\
\text { services }\end{array}$ & $\begin{array}{l}\text { International } \\
\text { Journal of } \\
\text { Bank } \\
\text { Marketing }\end{array}$ & 103 \\
\hline $\begin{array}{l}\text { LIANG; } \\
\text { WANG }\end{array}$ & $2007 b$ & $\begin{array}{l}\text { The behavioral sequence of information } \\
\text { education services industry in Taiwan: } \\
\text { relationship bonding tactics, relationship } \\
\text { quality and behavioral loyalty }\end{array}$ & $\begin{array}{l}\text { Measuring } \\
\text { Business } \\
\text { Excellence }\end{array}$ & 38 \\
\hline $\begin{array}{l}\text { LIANG; } \\
\text { WANG }\end{array}$ & $2007 a$ & $\begin{array}{l}\text { An insight into the impact of a retailer's } \\
\text { relationship efforts on customers' } \\
\text { attitudes and behavioral intentions }\end{array}$ & $\begin{array}{l}\text { International } \\
\text { Journal } \\
\text { Bank of } \\
\text { Marketing }\end{array}$ & 36 \\
\hline
\end{tabular}

Fonte: Elaborado pelos autores (2020).

\subsection{ANÁLISE QUALITATIVA DOS ARTIGOS}

Com base nas obras de maior relevância, citadas no Quadro 2, foi realizada uma breve análise sobre as mesmas com o propósito de expor o que os autores encontraram em suas pesquisas.

$\mathrm{Na}$ pesquisa de Liang e Wang (2006), é desenvolvido e testado empiricamente um modelo para examinar as relações entre as escalas de qualidade de serviço, táticas vinculantes de relacionamento, satisfação do cliente, confiança, comprometimento e lealdade de comportamento do cliente em um sistema de marketing de relacionamento. O objetivo principal dos autores foi testar se os consumidores consideravam construir relacionamentos de longo prazo com provedores de serviço com base em uma única instância de satisfação da qualidade de serviço. Os resultados obtidos pelos autores mostraram que nem todas as escalas tiveram efeito positivo sobre a qualidade de serviço percebida e em cada um 
dos três setores da empresa pesquisada, que foi um grande banco de Taiwan, as táticas vinculantes de relacionamento, que geram efeito positivo no relacionamento e retenção do cliente, foram diferentes: no setor de empréstimos e financiamentos o único vínculo de impacto foi o vínculo social, no setor de investimentos apenas o vínculo financeiro gerou impacto e no setor de cartão de crédito a tática de maior impacto foi a de vínculo social. A conclusão dos pesquisadores versou sobre a importância de gerenciar diferentes programas de marketing de relacionamento para cada segmento de clientes, pois só é possível alcançar resultados eficientes aplicando a tática certa para o cliente certo.

No estudo de Liang, Chen e Wang (2008), os autores objetivaram sua pesquisa empírica para testar um modelo que examina as relações entre táticas de vínculo de relacionamento, investimento no relacionamento percebido, qualidade do relacionamento percebida, fidelidade do cliente, retenção do cliente e compracruzada no contexto de consumidores de uma empresa de valores mobiliários online. Esse foi um dos poucos trabalhos que aborda a prática do marketing de relacionamento em empresas de serviços financeiros on-line, por isso da sua grande relevância acadêmica e prática. Os resultados desse estudo concluíram que apesar de existirem muitas maneiras de empresas on-line se engajarem nos relacionamentos com seus clientes, três táticas de vínculo são as mais bemsucedidas: vínculo financeiro, estrutural e social. Apontaram que através dos efeitos intervenientes do investimento em relacionamento percebido e qualidade percebida no relacionamento, todas as três táticas vinculantes aumentam a fidelidade do cliente, o que é de fato crucial para a melhoria na retenção de clientes e da compra cruzada. Os autores sugerem que os gerentes das empresas de serviços financeiros on-line devem alocar mais recursos para gerar vínculo social e estrutural do que para o financeiro, porque para tornar-se um cliente transacional em relacional a qualidade do serviço oferecido tem maior importância do que o preço.

Liang, Wang e Farquhar (2009) desenvolveram e testaram empiricamente um modelo conceitual no intuito de examinar a potencial influência dos investimentos em relacionamentos sobre atitude e comportamento do consumidor e a relação entre esses investimentos e o desempenho financeiro do cliente e da empresa. O estudo, realizado em um grande banco comercial asiático, apresentou resultados que revelam que a confiança do cliente, na honestidade no provedor, e o comprometimento aumentará quando o cliente estiver satisfeito com o investimento no relacionamento feito pelo provedor de serviços. Foi constatado que o investimento na satisfação do cliente exerce um efeito positivo no comportamento de compra. Os autores sugerem, que para adquirir resultados eficientes e excelente desempenho financeiro, às empresas devem realizar de forma simultânea diferentes programas de marketing para lidar com clientes de segmentações diferentes, pois são influenciados pelas táticas de vínculo.

Liang e Wang (2007b) testaram empiricamente um modelo que examina as relações entre táticas de relacionamento, investimentos em relacionamento percebido, satisfação do cliente, confiança, comprometimento e comportamento de lealdade do consumidor como variáveis controladas em um sistema de marketing de relacionamento. A pesquisa realizada, em uma escola de informática do maior instituto de serviços de informação de Taiwan, mostrou que os três tipos de táticas de vínculo de relacionamento têm influência significativa sobre o investimento em relacionamentos percebidos, exceto os financeiros. Além disso, os resultados mostraram que a satisfação do cliente é muito importante para reforçar as demais 
variáveis estudadas dentro do sistema de marketing de relacionamento. Os autores concluíram que não apenas as táticas de vínculo social, mas também as de vínculo estrutural têm efeitos positivos sobre o investimento no relacionamento percebido $\mathrm{e}$ na satisfação do cliente. Como sugestão, aos empreendedores, foi apresentada a máxima que a interação com o cliente e o fornecimento de serviços sob medida são as formas mais eficazes de garantir relacionamento com os clientes, além das já conhecidas soluções estruturais e serviço de valor agregado.

No estudo de Liang e Wang (2007a), os autores testaram um modelo empiricamente para examinar o impacto de diferentes esforços de relacionamento, feitos por um varejista, nos principais constructos do marketing de relacionamento. Os resultados apresentados indicam que os varejistas que realizam esforços de relacionamento com clientes fiéis afetam positivamente as suas atitudes e intenções comportamentais futuras. Os pesquisadores alegam que as equipes de trabalho precisam ser treinadas e motivadas a fazer esforços com clientes regulares, pois sua fidelidade é essencial para a sustentabilidade do negócio. E por fim, concluem que as três táticas de vínculo exercem um papel fundamental no relacionamento com o cliente de modo que afetam positivamente a satisfação do cliente, sua confiança e comprometimento, que por sua vez influenciam as intenções comportamentais do cliente, como permanecer na relação, por exemplo.

Com as análises foi possível identificar, nos 20 artigos sobre táticas vinculantes, que todas as pesquisas convergem para o mesmo resultado: táticas vinculantes de relacionamento impactam diretamente na satisfação, confiança, comprometimento e fidelização do cliente, inclusive, no comportamento e intenção futura.

\subsection{DISCUSSÃO DOS RESULTADOS}

Através dos resultados da pesquisa realizada foi percebido sua importância científica para o marketing de relacionamento, nos principais periódicos e bases de dados, iniciou em 2006 e que seu auge de publicação foi na década seguinte, em 2016. Nas empresas, conforme Milan, Eberle e Nespolo (2016), a manutenção e a ampliação das trocas relacionais reforçam a retenção de clientes e torna-se inclusive um diferencial frente à concorrência. Conforme Crescitelli e Silva (2015), cabe às empresas verificar se há um conjunto integrado de estratégias e ferramentas que estejam alinhadas para gerir os vínculos com o cliente.

Ao fornecer de táticas de vínculo de relacionamento de alto nível, Liang e Wang (2007b), afirmam que uma empresa pode consolidar seu relacionamento com os clientes de tal forma, que se essas táticas de vínculo forem integradas, será muito difícil para os concorrentes entrarem em seu mercado. No que tange o impacto das táticas de vínculo as variáveis mais pesquisadas foram: confiança, comprometimento, fidelidade, lealdade, satisfação, retenção de clientes, desempenho financeiro, intenção de recompra, comportamento, qualidade de serviço, atitude, custos de troca, envolvimento, valor percebido e duração do relacionamento.

$\mathrm{Na}$ maioria dos artigos os autores realizaram métodos de pesquisa quantitativa e alguns utilizaram quantitativa e qualitativa no mesmo estudo em etapas diferentes. Para análise dos modelos e verificação das escalas a maioria dos pesquisadores utilizou modelagem de equações estruturais. 
Em todos os artigos pesquisados, as táticas vinculantes influenciavam no investimento de relacionamento percebido, na qualidade do relacionamento cliente e empresa, na confiança, no comprometimento e na retenção. As empresas que investem em relacionamentos não apenas mantêm e estreitam as relações com seus clientes, como também atraem novos clientes (BERRY, 2002).

Os resultados desse estudo contribuíram de forma consistente para analisar conceitos e conteúdos sobre as táticas vinculantes. Ao mapear todos os construtos encontrados na pesquisa bibliométrica, compreendê-los e analisá-los foi possível identificar que eles são requisitos fundamentais para a manutenção de um marketing de relacionamento efetivo e sustentável, uma vez que tais táticas determinam a geração de valor percebido pelo cliente. $O$ valor ou valor percebido, segundo Milan at al. (2010), é o principal esteio dos relacionamentos existentes entre uma empresa e seus clientes, pois tem como função representar aquilo que o cliente entende, em um dado momento, como valioso para o início e para a manutenção de um relacionamento duradouro.

O futuro das táticas vinculantes estará entrelaçado ao futuro do marketing de relacionamento, que só tende a crescer e evoluir em forma de estratégia fundamental para o desempenho financeiro sustentável das empresas.

desenvolvimento de tais vínculos permitirá que as empresas de serviços alcancem uma diferenciação nos mercados onde estarão inseridas, permitindo que obtenham possíveis vantagens competitivas sustentáveis, melhorando o seu desempenho econômico-financeiro (DAVCIK; SHARMA, 2016).

\section{CONSIDERAÇÕES FINAIS}

As táticas vinculantes de relacionamento impactam diretamente na satisfação, confiança, comprometimento e fidelização do cliente, inclusive, no comportamento e intenção futura. Todas as três táticas promovem o relacionamento do cliente com o negócio por meio dos aspectos já citados anteriormente. Cada tática deve ser usada de acordo com o tipo de serviço realizado e com os clientes que o negócio possui, nem sempre é possível aplicar as três táticas simultaneamente.

A importância desse estudo se deve ao seu ineditismo, pois não há nenhum registro de publicação que tenha feito análise bibliométrica e mapeado de forma exploratória as obras sobre táticas vinculantes. Observa-se que existem uma quantidade pequena de estudos sobre as táticas vinculantes. Como elas são importantes para o relacionamento com o cliente, elas deveriam ser mais abordadas pela literatura e serem mais pesquisadas porque isso mostraria que este construto é recém abordado, relevante e o quanto ele pode influenciar não apenas na questão do relacionamento, mas na retenção e fidelização do cliente

Um campo e gap de pesquisa a ser explorado foi como apenas uma das táticas vinculantes poderiam ser pesquisadas em diferentes segmentos da área de serviços. Os estudos nos apresentaram todas as táticas relacionadas aos variados aspectos do relacionamento com o cliente. Outro apontamento está em como cada tática vinculante podem influenciar individualmente cada aspecto do relacionamento como a lealdade e o comprometimento do cliente.

Como limitações do estudo, não houve uma restrição maior de áreas de pesquisa na análise bibliométrica como o foco na área de gestão e negócios com relação direta com 0 marketing. Para estudos futuros, deve-se continuar com 
pesquisas que visem estimular análises dos modelos propostos, testar e comparar modelos alternativos, inclusive em diferentes ambientes para que se evolua na área teórica e empírica as práticas das táticas vinculantes como estratégias do marketing de relacionamento efetivo.

Agradecimentos: Este estudo possui fomento da CAPES/FAPERGS e CAPES/PROSUC.

\section{REFERÊNCIAS}

ARANTOLA, H. Consumer Bonding: A Conceptual Exploration. Journal of Relationship Marketing, v. 1, p. 93-107, 2002.

BERNARDON, P. S.; LOPES, E. L. Os antecedentes da lealdade em um contexto de crise econômica: um estudo de caso em uma loja de roupas infantis. Revista de Administração, v. 16, n. 1, p. 121-150, 2018.

BERRY, L. Relationship marketing. Emerging perspectives of services marketing (Proceedings Series). Chicago: Amer Marketing Assn, 1983.

BERRY, L. L. Relationship marketing of services - Growing interest, emerging perspectives. Journal of the Academy of Marketing Science, v. 23, n. 4, p. 236245, 1995.

BERRY, L. L. Relationship marketing of services: perspectives from 1983 and 2000. Journal of Relationship Marketing, Binghanton, v. 1, n. 1, p. 59-77, 2002.

BERRY, L.L. PARASURAMAN, A. Marketing services: Competing through quality. New York: The Free Press, 1991.

BURNHAM, T. A.; FRELS, J. K.; MAHAJAN, V. Consumer switching costs: a typology antecedentes, and consequences. Journal of the Academy of Marketing Science, v. 31, p. 109- 126, 2003.

CHIU, H.-C.; HSIEH, Y. C.; LI, Y. C.; LEE, M. Relationship marketing and consumer switching behavior. Journal of Business Research, v. 58, n. 12, p. 1681-1689, 2005.

CRESCITELLI, E.; SILVA, S. S. Marketing de Relacionamento para o Público de Baixa Renda: Estudo Exploratório no Varejo de Eletrodomésticos. Revista de Administração, v. 13, n. 3, p. 28-54, 2015.

DAVCIK, N.; SHARMA, P. Marketing resources, performance, and competitive advantage: A review and future research directions. Journal of Business Research, v. 69, n. 12, p. 5547-5552, 2016. 
DONG, D.-H.; ZHANG, R.-X.; YANG, G. A Reexamination and Extension of Customer Bonding Tactics in B2C E-Commerce Industry. In: 2009 International Conference on Management and Service Science. IEEE, 2009. p. 1-4.

EDWARD, M.; SAHADEV, S. Role of switching costs in the service quality, perceived value, customer satisfaction and customer retention linkage. Asia Pacific Journal of Marketing and Logistics, v. 23, n. 3, p. 327-345, 2011.

FIGUEIREDO, N. Tópicos modernos em Bibliometria. Brasília: Associação dos Bibliotecários do Distrito Federal, 1977.

HALIM, R. E. The economic impact of relationship bonding tactics: a research agenda. European Research Studies Journal. v. 21, n. 1, p. 65-81, 2018.

HAN, S.-L. Antecedents of buyer-seller long-term relationships: An Exploratory model of structural bonding and social bonding: (Doctoral dissertation). The Pennsylvania State University, 1991.

HENNIG-THURAU, T.; GWINNER, K. P.; GREMLER, D. D. Undestanding relationship marketing outcomes: an integration of relational benefits and relationship quality. Journal of Service Research, v. 4, n. 3, p. 230-247, 2002.

HENNINK, M.; HUTTER, I.; BAILEY, A. Qualitative research methods. Thousand Oaks: Sage Publications, 2011.

HSIEH, Y.-C.; CHIU, H.-C.; CHIANG, M.-Y. Maintaining a committed online customer: A study across search-experience-credence products. Journal of Retailing, v. 81, n. 1, p. 75-82, 2005.

JONES, M. A.; MOTHERSBAUGH, D. L.; BEATTY, S. E. Switching barriers and repurchase intentions in services. Journal of Retailing, v. 76, n. 2, p. 259-274, 2000.

KOTLER, P.; KELLER, K. L. Administração de marketing. 14. ed. São Paulo: Pearson Prentice Hall, 2012.

KOUL, S.; SINHA, P. K.; MISHRA, H. G. Antecedents to customer dependency in buyer- seller relationship: A BOP retailer investigation. Global Business Review, v. 1, n. 3, p. 610-629, 2016.

LAKATOS, E. M.; MARCONI, M. de A. Metodologia científica. 6. ed. São Paulo: Atlas, 2011.

LAKSAMANA, P.; WONG, D.; KINGSHOTT, R. P.; MUCHTAR, F. The role of interaction quality and switching costs in premium banking services. Marketing Intelligence \& Planning, v. 31, n. 3, p. 229-249, 2013.

LEE, S. Y. Analysis of relationship marketing factors for sports centers with mixed methods research. Asia Pacific Journal of Marketing and Logistics, v. 30, n. 1, p. 182-197, 2018. 
LEENHEER, J.; BIJMOLT, T. H. A. Which retailers adopt a loyalty program? An empirical study. Journal of Retailing and Consumer Services, v. 15, n. 6, p. 429442, 2008.

LIANG, C-J.; CHEN, H.-J.; WANG, W.-H. Does online relationship marketing enhance customer retention and cross-buying? Service Industries Journal, v. 28, n. 6, p. 769-787, 2008.

LIANG, C.-J.; WANG, W.-H. The behavioural sequence of the financial services industry in Taiwan: Service quality, relationship quality and behavioural loyalty. Service Industries Journal, v. 26, n. 2, p. 119-145, 2006.

LIANG, C.-J.; WANG, W.-H. An insight into the impact of a retailer's relationship efforts on customers' attitudes and behavioral intentions. International Journal of Bank Marketing, v. 25, n. 5, p. 336-366, 2007a.

LIANG, C.-J.; WANG, W.-H. The behavioral sequence of information education services industry in Taiwan: relationship bonding tactics, relationship quality and behavioral loyalty. Measuring Business Excellence, v. 11, n. 2, p. 62-74. 2007b.

LIANG, C-J; WANG, W.-H. How managers in the financial services industry ensure financial performance. Service Industries Journal, v. 28, n. 2, p. 193-210, 2008.

LIANG, C-J; WANG, W.-H.; FARQUHAR, J. D. The influence of customer perceptions on financial performance in financial services. International Journal of Bank Marketing, v. 27, n. 2, p. 129-149, 2009.

LIN, N.-P; WENG; JAMES, C. M.; HSIEH, Y.-C. Relational bonds and customer's trust and commitment-a study on the moderating effects of web site usage. Service Industries Journal, v. 23, n. 3, p. 103-124, 2003.

LOPES, E. L.; FURRIER, M. T.; PIZZINNATTO, N. K.; CUNHA, R. Táticas vinculantes em Marketing de Relacionamento: uma aplicação no varejo tradicional e virtual. Revista Base - Revista de Administração e Contabilidade da Unisinos, v. 9, n. 2, p. 116-133, 2012.

MADHOK, A. Revisiting multinational firms tolerance for joint ventures: A trust-based approach. Journal of International Business Studies, v. 26, n. 3, p. 117-137, 1995.

MERTON, R. K. The Mathew effect in science. Science, v. 159, n. 3810, p. 58, Jan. 1968.

MILAN, G. S.; RIBEIRO, J. L. D.; DE TONI, D.; LARENTIS, F. A prática relacional entre provedor de serviços e clientes: comparação entre um modelo teórico e um modelo rival. Revista Base - Revista de Administração e Contabilidade da Unisinos, v. 7, n. 1, p. 42-54, 2010. 
MILAN, G. S.; EBERLE, L.; NESPOLO, D. Determinantes da retenção de clientes: um estudo comparativo em dois ambientes de prestação de serviços. Revista Base - Revista de Administração e Contabilidade da Unisinos, v. 13, n. 3, p. 216-234, 2016.

MILAN, G. S.; SLONGO, L. A.; EBERLE, L.; DE TONI, D.; BEBBER, S. Determinants of customer loyalty: a study with customers of a Brazilian bank. Benchmarking: an International Journal, v. 25, n. 9, p. 3935-3950, 2018.

MIMOUNI-CHAABANE, A.; VOLLE, P. Perceived benefits of loyalty programs: Scale development and implications for relational strategies. Journal of Business Research, v. 63, n. 1, p. 32-37, 2010.

NARTEH, B.; AGBEMABIESE, G. C.; KODUA, P.; BRAIMAH, M. Relationship marketing and customer loyalty: Evidence from the Ghanaian luxury hotel industry. Journal of Hospitality Marketing \& Management, v. 22, n. 4, p. 407-436, 2013.

OLIVER, R. L. Satisfaction: a behavioral perspective on the consumer. 2. ed. New York: M. E. Sharpe, 2010.

PALMATIER, R. W.; DANT, R. P.; GREWAL, D.; EVAN, K. R. Factors influencing the effectiveness of relationship marketing: a meta-analysis. Journal of Marketing, $\mathrm{V}$. 70, n. 4, p.136-153, 2006.

PARAHOO, S. K. Credit where it is due: drivers of loyalty to credit cards. International Journal of Bank Marketing, v. 30, n. 1, p. 4-19, 2012.

PARASURAMAN, H; ZEITHAML, S. A comparison of service quality in the banking industry: some evidence from Turkish - and Greek - speaking areas in Cyprus. International Journal of Bank Marketing, v. 23, n. 7, p. 508-526, 2005.

PAULSSEN, M.; ROULET, R.; WILKE, S. Risk as moderador of the trust-loyalty relationship. European Journal of Marketing, v. 48, n.5/6, p. 964-981, 2014.

PELTIER, J. W.; WESTFALL, J. Dissecting the HMO-benefits managers relationship: what to measure and why. Marketing Health Services, v. 20, n. 2, 2000.

RODRIGUEZ, C. M.; WILSON, D. T. Relationship bonding and trust as a foundation for commitment in U.S.-Mexican strategic alliances: A structural equation modeling approach. Journal of International Marketing, v. 10, n. 4, p. 53-76, 2002.

SHI, G.; BU, H.; PING, Y.; LIU, M. T.; WANG, Y. Customer relationship investment and relationship strength: evidence from insurance industry in China. Journal of Services Marketing, v. 30, n. 2, p. 201-211, 2016.

TURNER, R. H. Family interaction. John Wiley \& Sons, 1970.

VAVRA, T. G. Vender após a venda: as vantagens do aftermarketing. Supervisão, v. 55, n. 10, pág. 9-12, 1994. 
VERHOEF, P. C. Understanding the effect of customer relationship management efforts on customer retention and customer share development. Journal of marketing, v. 67, n. 4, p. 30-45, 2003.

WANG, W.-H. The interrelationship of retailer's relationship efforts and consumers' attitude and behavior. Measuring Business Excellence, v. 12, n. 1, p. 13-28, 2008.

WANG, W.-H.; LIANG, C. Consumer behavior of the information services industry in Taiwan - conceptual framework and hypotheses development. Measuring Business Excellence, v. 14, n. 2, p. 12-21, 2010.

WANG, W.; LIANG, C.; WU, Y. Relationship bonding tactics, relationship quality and customer behavioral loyalty - behavioral sequence Taiwan's information services industry. Journal of Services Research, v. 6, n. 1, p. 345-356, 2006.

WANG, Y.; WANG, N.; JIANG, L.; YANG, Z; CUI, V. Managing relationships with power advantage buyers: The role of supplier initiated bonding tactics in long-term buyer-supplier collaborations. Journal of Business Research, v. 69, n. 12, p. 55875596, 2016.

WANG, Y.-S. Buyer-seller relationships of the dental devices industry in Taiwan: perspective of relationship. Journal of Business-to-Business Marketing, v. 25, n. 3, p. 169-186, 2018.

WEI, K.; CROWSTON, K.; LI, N. L.; HECKMAN, R. Understanding group maintenance behavior in free/Libre open-source software projects: the case of fire and Gaim. Information \& Management, v. 51, n. 3, p. 297-309, 2014.

WILSON, D. T.; VENKATAPPARAO, M. Bonding and Commitment in Buyer-Seller Relationships: A Preliminary Conceptualisation. Industrial Marketing and Purchasing, v. 1, n. 3, p. 44-58, 1986.

WULF, K.; ODEKERKEN-SCHRÖDER, G.; IACOBUCCI, D. Investments in consumer relationships: a cross-country and cross-industry exploration. Journal of Marketing, v. 65, n.4, p. 33-50, 2001.

YUFANG, J.; XIAOBING, S. Bonding Tactic Sets of On-Line Stores: An Empirical Study. Joint Conference on Service Sciences, v. 34, n. 4, p. 95-99, 2012.

ZHANG, J. Channel Coordination in the Presence of a Dominant Retailer. Marketing Science, v. 24, n. 2, p. 254-262, 2005.

ZHANG, J.; DONG, H.; ZHANG, J. Understanding customer satisfaction and loyalty: An empirical study of mobile instant messages in China. International Journal of Information Management, v. 30, n. 4, p. 289-300, 2010. 